EOMmun Communication et organisation

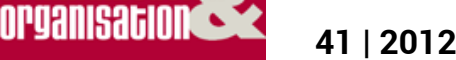

La mutation du métier de communicant public

\title{
Les plateformes électroniques de services
}

Quels enjeux pour les responsables de communication territoriale?

Jean-Baptiste Le Corf

\section{CpenEdition}

Journals

Édition électronique

URL : http://journals.openedition.org/communicationorganisation/3755

DOI : 10.4000/communicationorganisation.3755

ISSN : 1775-3546

Éditeur

Presses universitaires de Bordeaux

Édition imprimée

Date de publication : 1 juin 2012

Pagination : 101-112

ISBN : 978-2-86781-821-9

ISSN : $1168-5549$

Référence électronique

Jean-Baptiste Le Corf, «Les plateformes électroniques de services », Communication et organisation

[En ligne], 41 | 2012, mis en ligne le 01 juin 2015, consulté le 10 décembre 2020. URL : http://

journals.openedition.org/communicationorganisation/3755 ; DOI : https://doi.org/10.4000/

communicationorganisation.3755 


\title{
Les plateformes électroniques de services : quels enjeux pour les responsables de communication territoriale?
}

\author{
Jean-Baptiste Le Corf ${ }^{1}$
}

Dans un contexte de massification des usages du web 2.0, les villes témoignent d'un intérêt croissant pour les plateformes numériques de services aux habitants. Si ces dispositifs sont d'abord utilisés pour moderniser les formes de la gestion publique locale, les politiques de communication territoriale sont aussi affectées par l'introduction de ces plateformes (Le Corf, 2011). Ces initiatives donnent ainsi l'occasion aux villes de communiquer à nouveau autour de la " participation » et de la " proximité ", deux notions qu'il convient de distinguer. La participation peut être définie, au sens large, comme " un ensemble d'activités par lesquelles les citoyens sont habilités à entrer en contact avec l'univers du pouvoir en respectant certaines contraintes » (Krzątała-Jaworska, 2012), tandis que la notion de proximité est entendue dans une acception plus gestionnaire et peut renvoyer, comme l'observe Loïc Blondiaux (2008, p. 20), à la dimension managériale de la " cité par projets " (Boltanski et Chiapello 1999). Ces deux notions sont étroitement liées lorsque la "participation » implique un transfert de pouvoir aux habitants sous la forme d'un partenariat très encadré, permettant d' " associer des citoyens en tant qu'usagers à la gestion, voire la coproduction des services publics» (Bacqué, Rey, Sintomer 2005 : 17). Le paradigme de la " proximité ", marqué par la référence au "savoir d'usage " des citoyens, est défendu dans des "optiques auto-gestionnaires ", dans la continuité des réformes s'inscrivant dans l'esprit du New Public Management (Sintomer 2008), qui postule que la modernisation doit se tourner vers les citoyens et s'appuyer sur leur apport pour optimiser l'efficacité des services rendus. Cette logique managériale, qui peut être instrumentée avec des plateformes TIC, s'adresse d'abord à un public local, invité à travers différentes opérations

1 Doctorant en sciences de l'information et de la communication sous la direction de Philippe Bouquillion et ATER à l'université Paris 8. Membre du laboratoire CEMTI (centre d'études sur les médias, les technologies et l'internationalisation) à l'université Paris 8 ; jblecorf@club-internet.fr 
de communication territoriale à apporter sa contribution. Dans le cadre de cet article, nous analyserons la communication territoriale portant sur une plateforme d'échanges de services entre habitants, rattachée à différents sites Internet municipaux. Le fait que ce dispositif soit développé par un même prestataire (une agence web qui vend un service aux collectivités territoriales), nous permet d'analyser les pratiques professionnelles des responsables de la communication de quelques villes clientes de ce prestataire. Nous choisissons de sélectionner les plateformes TIC municipales de notre corpus à partir de deux facteurs susceptibles d'impacter potentiellement la communication territoriale accompagnant la mise en place de cette plateforme : premièrement, la taille de la ville (mesurée par le nombre d'habitants), qui détermine souvent les moyens humains et financiers dont dispose le service communication de la municipalité ${ }^{2}$; deuxièmement, l'histoire de la politique de la ville en matière de $\mathrm{TIC}^{3}$. Toutes les villes de notre échantillon utilisent la même plateforme TIC qui apparaît comme un " réseau social local » où sur un fond de carte Google Maps, les habitants peuvent échanger des services par leurs centres d'intérêts ou par proximité géographique, comme le montre la copie d'écran ci-dessous illustrant le cas d'Issy-les-Moulineaux :
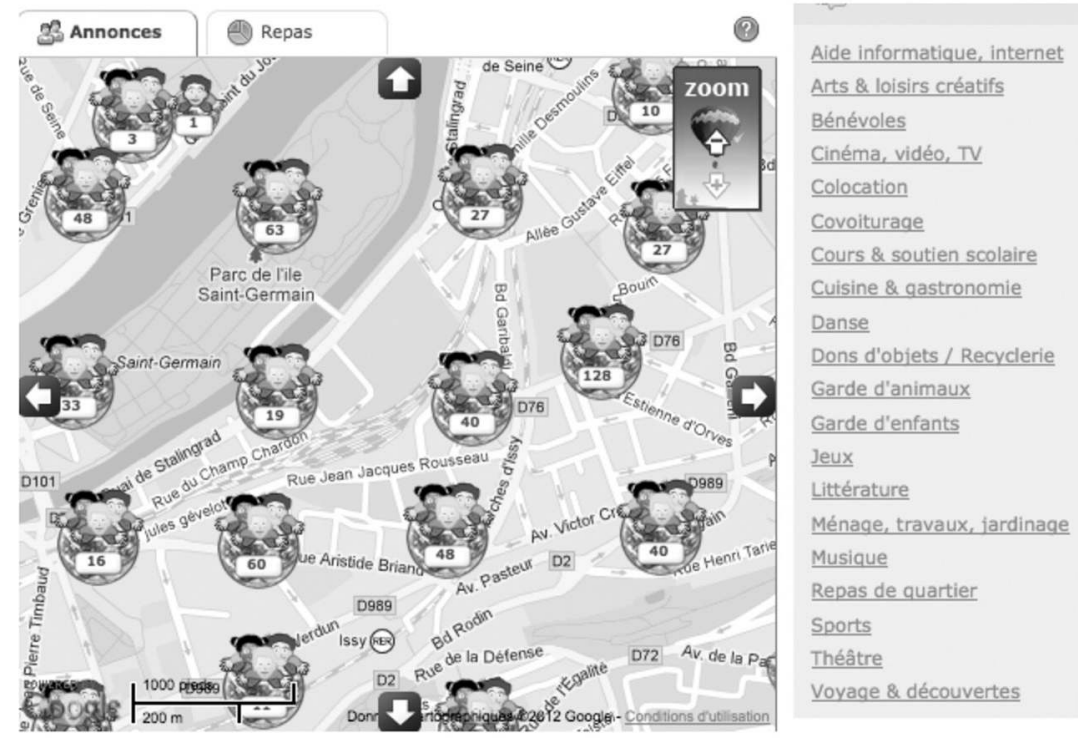

Plateforme d'échanges de services entre habitants d'Issy-les-Moulineaux

\footnotetext{
2 Dans notre corpus de réseaux sociaux municipaux, nous distinguons des villes de moins de 30000 habitants (Blagnac, Bois-Colombe, La Celle-Saint-Cloud, les Pennes-Mirabeau), d'autres entre 30000 et 60000 habitants (Nevers, Paris 6e, Poissy, Saint-Ouen), une ville de plus de 60000 habitants (Issy-les-Moulineaux). 3 Pour exemple, Issy-les-Moulineaux est réputée comme active en matière de participation des habitants en ligne et pionnière dans les expérimentations via les TIC, à l'inverse par exemple de Saint-Ouen. Le positionnement d'une ville vis-à-vis des TIC peut avoir des répercussions sur la communication territoriale concernant cette thématique.
} 
Deux éléments peuvent seulement varier en fonction des spécificités de chaque ville : le nom de la plateforme (reprenant le nom des habitants) et les fonctionnalités de la plateforme. Notre approche vise à observer les pratiques des responsables de communication des villes utilisant ce dispositif au regard de certains travaux qui se sont intéressés à la professionnalisation de la communication locale (Fourdin 1994 ; Pailliart 2006 notamment). Pour comprendre comment les politiques de communication des villes et les pratiques des communicants publics sont affectées par l'introduction de ce dispositif, nous montrerons dans un premier temps, à partir de l'analyse des sites Internet des municipalités mettant en place cette plateforme (2009) et de certaines actions de communication publique, de quelles manières les communicants publics cherchent à garantir et à renouveler une information pour communiquer sur le nouveau service offert aux habitants. Dans un deuxième temps, en nous appuyant sur l'analyse d'un corpus d'articles portant sur la plateforme parus dans les bulletins municipaux (2008 et 2009) et sur l'analyse d'entretiens semi-directifs menés en mars et avril 20094. Nous verrons comment les professionnels des services communication des villes conservent une autonomie relativement limitée vis-à-vis de l'instance politique et comment les pratiques en matière de communication politique sont ainsi renouvelées. Dans un troisième temps, en confrontant l'analyse du dispositif à l'analyse des entretiens, nous analyserons l'élargissement des compétences du responsable de communication, au-delà des aspects techniques, et sa nouvelle position de stratège qui découle de l'adoption de cette plateforme dans les villes. En conclusion, nous prolongerons notre problématique en nous interrogeant sur l'importance accordée à la communication territoriale pour atteindre les objectifs de management public local reposant sur des plateformes TIC et sur la visée plus opérationnelle associée à la communication territoriale pour répondre à ces enjeux. Ces mutations de la communication territoriale se retrouvent plus encore aujourd'hui avec les plateformes de données publiques et de services numériques qui ne cessent de se développer avec le mouvement « open data».

\section{La communication sur la plateforme : l'information aux habitants}

Les villes ont besoin de faire connaître la plateforme aux habitants. Il s'agit de montrer que ce nouveau service peut potentiellement répondre à tous leurs besoins au quotidien. L'analyse des sites Internet municipaux (SIM) de notre échantillon montre que, dans la plupart des cas, les professionnels créent de deux façons des accès à cette plateforme, d'une part en insérant un logo permanent (qui permet de représenter la plateforme et montre son ancrage dans la communication générale de la ville) et, d'autre part, en faisant un

4 Nous avons choisi de mettre ici en perspective trois entretiens menés auprès des directions de la communication d'Issy-les-Moulineaux et de Poissy, ainsi qu'auprès de la webmestre de Saint-Ouen en raison des deux facteurs mentionnés ci-dessus (taille de la ville et histoire de sa politique TIC). 
renvoi contextualisé (qui permet de valoriser un type de service mis en place dans cette plateforme). Cet exemple du SIM de Nevers est symptomatique de ces deux types d'accès :

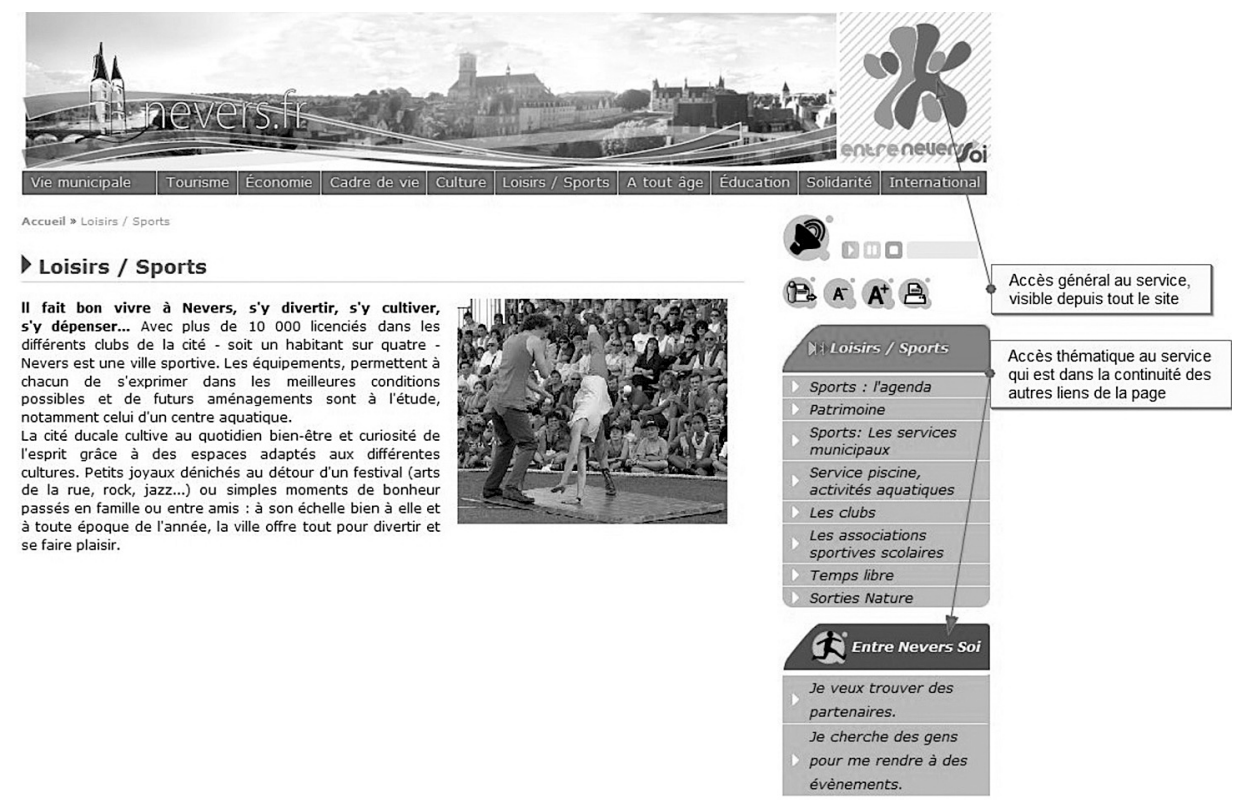

Deux types d'accès à la plateforme à partir de la page "sports et loisirs " du SIM de Nevers

Ces deux accès à la plateforme sont récurrents dans les SIM que nous avons analysés. En complément d'un accès général à la plateforme, le responsable du site Internet effectue souvent une communication contextualisée à partir des pages thématiques existantes du SIM (culture, enfance, sport, logement...) qui peuvent renvoyer, selon les cas, à différents usages et fonctionnalités de la plateforme (spectacles, gardes d'enfants, trouver un partenaire de sport, colocation...). Cette standardisation des pratiques professionnelles des responsables des SIM dans la valorisation de cette plateforme peut s'expliquer par le fait que ces derniers reçoivent des conseils de la part de leur prestataire, qui leur propose aussi un « accompagnement » en matière de communication, comme nous le montrons par ailleurs (Le Corf, 2012).

En dehors du SIM, notre analyse d'un corpus d'actions de communication publique portant sur la plateforme montre que les responsables de la communication peuvent aussi informer les habitants sur la nouvelle plateforme offerte par d'autres canaux, par exemple via une campagne d'affichage public sur des panneaux JCDecaux, comme l'illustre notamment l'exemple ci-après : 
Dans cet exemple, la campagne de communication qui utilise le symbole de la montgolfière promeut en ce sens l'image d'un habitant autonome et qui a une vision en surplomb $\mathrm{du}$ territoire. Toutes ces actions de communication, visant à faire connaître cette plateforme d'échanges de services, pourraient s'interpréter comme une volonté des villes de fournir des services plus adéquats (c'est-à-dire répondant mieux à la diversité des besoins de chacun) grâce à la participation des usagers. Si cette logique de la collectivité comme prestataire de services n'est pas nouvelle, il faut remarquer que les politiques de communication jouent ici un rôle primordial pour renforcer cette dynamique souhaitée par les responsables politiques des villes.

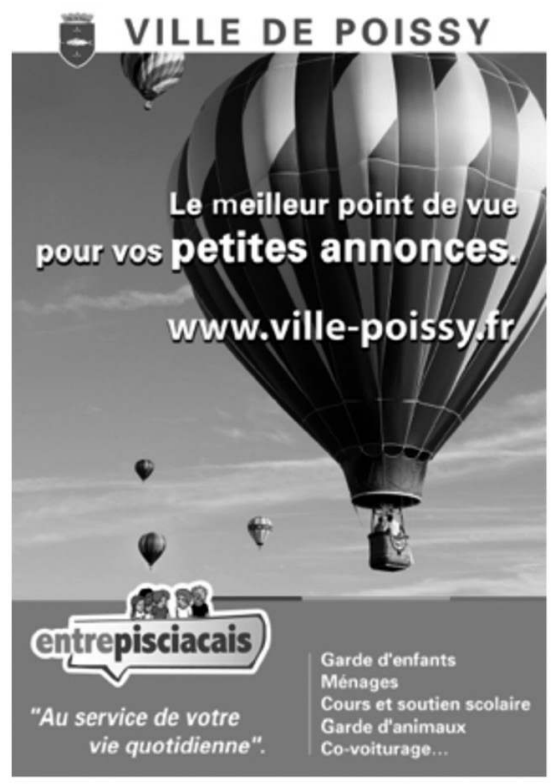

Campagne d'affichage public de Poissy sur des panneaux Decaux en septembre 2007 après le lancement de la plateforme de mai 2007

\section{Nouvelles pratiques en matière de communication politique et relation historique avec l'instance politique}

Notre analyse de la communication politique produite par les communicants publics s'inscrit dans le prolongement des rapports étroits qui existent entre les professionnels du service communication et l'instance politique (Pailliart 2006). Le thème de la « convivialité » et la «rhétorique de l'efficacité » ressortent tout particulièrement dans la communication territoriale accompagnant la mise en place de cette plateforme dans les villes.

\section{La convivialité et l'entraide, des référents identitaires associés à des objectifs politiques}

La stratégie des communicants, visant à créer un sentiment d'appartenance des habitants à une même communauté pour accroître leur participation, passe d'abord par le nom des plateformes qui, dans la plupart des cas, insistent sur l'appartenance des habitants à un collectif : «Entre Pisciacais ", «Entre Blagnacais ", "Entre Bois-Colombiens », "Entre Nevers soi », "Entre Conflanais », «Entre Pennois ». Cette « rhétorique de la proximité », utilisée pour ses capacités supposées à produire du lien social (Nonjon 2005:171), a une visée opérationnelle car elle vise à accroître la participation des habitants, laquelle est un préalable au fonctionnement de ce type de plateforme 
d'échanges de services. Elle s'apparente aussi à communication sociale servant à " modifier les représentations, les comportements ou à renforcer les solidarités » (Lavigne 2008:239), et utilisée pour stimuler cette participation visant à mieux répondre, dans une optique gestionnaire, aux problèmes de la vie quotidienne. Cette visée opérationnelle de la communication publique territoriale entretient un lien étroit avec une communication politique qui met surtout l'accent sur deux aspects : les potentialités du dispositif à (re)créer du lien social sur le territoire et le soutien de la ville en faveur des TIC.

Premièrement, la plateforme, médiation technique liée à la vie locale de quartier, est en effet présentée comme le lieu du développement social urbain. Cette idée est d'ailleurs présente dans la plupart des discours tenus par les journalistes municipaux que nous avons analysés, comme l'illustrent notamment ces propos:

«Avec Entre Nevers soi » service de mise en relation des habitants, quiconque dispose d'une connexion internet pourra désormais partager, échanger, découvrir ou confronter ses talents, ses passions, ses envies avec d'autres [...]. Un service gratuit, simple comme un clic et qui va permettre de (re)tisser du lien social. Un carrefour de solidarité active et d'entraide interactif » (Magazine municipal de Nevers, septembre 2008)

Ces discours, insistant sur les potentialités du nouveau dispositif à «transformer le territoire » et que l'on retrouve dans les nombreux articles portant sur la plateforme au sein des bulletins municipaux des villes, sont à mettre en perspective avec des tendances de plus long terme de la communication territoriale. Ainsi, la communication sur la plateforme se comprend d'autant plus que le journal municipal intéresse les élus car il présente des enjeux politiques en s'imposant comme un outil de construction des référents identitaires (Pailliart 2006: 117) et de « mise en scène du lien social » (Ibid: 119). L'analyse des bulletins municipaux des villes de notre échantillon montre que, malgré la diversité des territoires, le bulletin municipal reste un lieu propice à l'utilisation des mêmes « totems » (Le Bart 2000:179) autour de la «solidarité/convivialité/entraide ». Cette communication montre que l'échelle du quartier (ici favorisée au sein du dispositif par une technologie de géolocalisation) est considérée comme idéale pour revitaliser la citoyenneté et l'identité locale, ainsi que pour favoriser la " gestion des choses simples " éloignées "de l'abstraction du politique ", ce qui évacue comme l'observe Catherine Neveu toute dimension de conflictualité (Neveu 2004: 64).

Deuxièmement, l'analyse des discours de la communication territoriale révèle que la thématique de l'entraide est associée au thème de la modernité via les TIC, qui est un autre invariant de la communication politique des collectivités territoriales depuis que celles-ci se sont intéressées aux TIC (Pailliart 2000). Cette dimension ressort bien dans ces propos notamment : 
«L'interactivité entre dans une nouvelle ère sur le site Internet de la ville de Nevers. Un service gratuit, simple comme un clic » (Magazine municipal de Nevers, septembre 2008)

Cet extrait de discours, représentatif de la recherche de légitimité des acteurs territoriaux, illustre bien aussi le fait que la communication territoriale vise à démontrer aux citoyens que la collectivité dispose des meilleurs services et surtout que ces services sont " efficaces".

\section{Une " rhétorique de l'efficacité "}

Les journalistes municipaux ont également recours à une "rhétorique de l'efficacité ", marquée par une quantification et une mise en scène de chiffres et d'indicateurs de performance, également récurrente au sein de la communication de l'État (Ogien 2009: 57). Ainsi, dans la plupart des articles faisant la promotion du dispositif au sein des bulletins municipaux, les statistiques d'utilisation sont souvent mentionnées, à l'instar de cet exemple :

"Ce site est fait pour vous. En un mois, 1500 visiteurs sont déjà recensés, plus de 80 profils ont été créés et quelque 40 annonces sont actives. Une visite s'impose !» (extrait d'un article du magazine municipal de Bois-Colombes, février 2009)

De surcroît, au-delà de la communication à destination des habitants, l'analyse des entretiens révèle d'ailleurs aussi qu'il existe un important effet de stimulation entre les communicants publics des différentes collectivités vis-à-vis des statistiques d'utilisation des autres collectivités utilisant la plateforme de services. Ce phénomène est lié à l'action du prestataire, qui met en concurrence ses villes clientes les unes par rapport aux autres, en faisant ces classements (Le Corf, 2012). Les communicants publics répondent à cette logique de "médiatisation de l'évaluation " qui les oblige à se positionner dans une dimension stratégique de valorisation de la plateforme :

«On est souvent $2^{\text {e }}$ derrière Puteaux [...]. On a environ entre 70 et 80 annonces créées. Je regarde ces statistiques. Mais il peut y avoir des baisses [...]. Quand je ne parle pas du service dans le journal, il y a une baisse mais dans l'ensemble on reste assez stable. Le but serait de trouver un pic pour doubler Puteaux, surtout qu'on a une perte de vitesse et que Saint-Maur remonte " (Directrice de la communication de Poissy)

À travers ce retour d'usages permettant d'évaluer l'importance de la figure du " contributeur » sur laquelle repose l'efficacité de ces nouveaux services urbains (Cormerais 2009), il s'agit de montrer les potentialités de la plateforme mise en place par la ville et sa capacité (et par extension celle des élus) à faciliter la vie des habitants au quotidien. Cette capacité devient en quelque sorte mesurable par l'activité sur la plateforme, tandis que l'observation des usages donne davantage un rôle de stratège aux responsables de la communication territoriale. 


\section{L'élargissement des compétences du responsable de communication : du technicien au stratège}

Notre observation montre qu'avec l'adoption de la plateforme, les compétences techniques attribuées aux communicants publics (Pailliart 2006) sont prolongées par des compétences en matière de gestion des services à la personne qui renforcent leur rôle stratégique à trois niveaux.

D'abord, cet élargissement des compétences repose en partie sur le dispositif qui permet aux communicants publics d'avoir une stratégie d'effacement visà-vis de cette plateforme dans la mesure où ce type de dispositif participatif en ligne dispose de formats de contribution spécifiques ne rendant pas possible les formes d'expressions plus contestataires ou polémiques. La production de l'annonce est très encadrée comme le montre la copie d'écran ci-dessous :

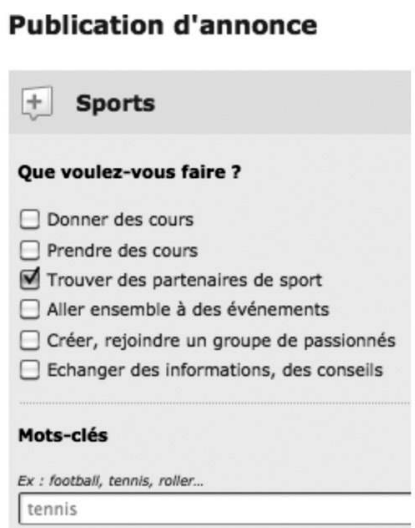

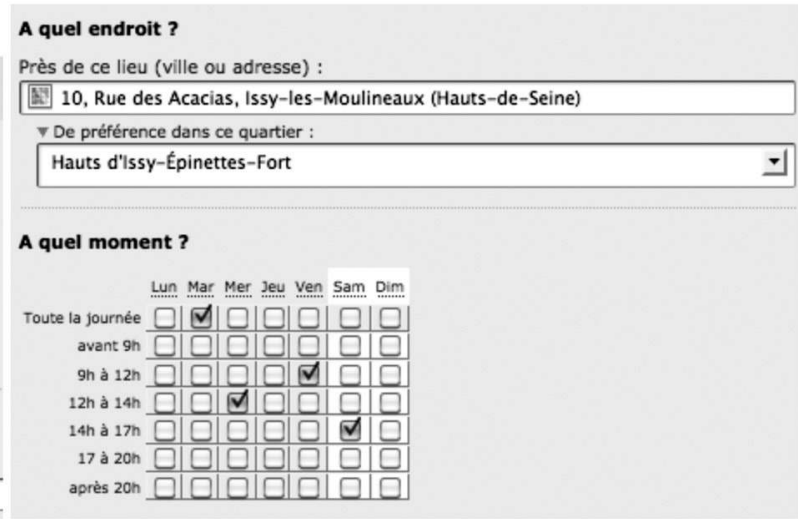

Exemples de formats de contribution pour publier une annonce dans la rubrique " sport » de la plateforme d'Issy-les-Moulineaux

Par conséquent, la nature même du dispositif permet au communicant public d'adopter une stratégie d'effacement vis-à-vis des utilisateurs, stratégie que l'on retrouve chez tous les responsables de communication rencontrés mais qui ressort particulièrement bien dans ces propos:

"J'ai un principe, c'est de ne pas contacter les utilisateurs [...] pour pas qu'ils aient l'impression que la mairie est derrière et les surveille [...], c'est pour faire comme si c'était un vrai réseau social de web 2.0 sans contrôle [...]. On veut être neutre [...]. On le considère comme une porte vers un service privé qui nous plait mais ce n'est pas celui de la ville et qui appartient aux habitants [...]. Notre rôle c'est juste de mettre cet outil à disposition des habitants » (Directeur du service communication d'Issy-les-Moulineaux).

Cette volonté d'être « neutre » (« c'est pour faire comme si ») peut faire échos aux «faux-semblants » de la transparence, qui peut être considérée comme une idéologie (Olliver-Yaniv 2003). 
Deuxièmement, le réseau, qui renvoie à cette notion de transparence, peut au contraire être instauré en une technologie de pouvoir (Musso 2003:55), puisque le dispositif permet - notamment aux responsables de communication - de mieux connaître les pratiques des administrés et de détenir des informations stratégiques grâce à la production d'une information locale ascendante par les habitants. Les communicants publics rencontrés ont manifesté leur intérêt de mieux connaître les échanges des habitants, ainsi que leurs attentes et besoins au quotidien pour mieux orienter les politiques locales. Dès lors, les réseaux sociaux institutionnalisés par les villes pourraient peut-être aussi être à reconsidérer dans la lignée d'autres dispositifs de surveillance qui participent des systèmes de contrôle des citoyens par une surveillance par les données (Mattelart, 2007).

Enfin, la communication territoriale (notamment les discours des bulletins municipaux que nous avons présentés) s'inscrit aussi dans une dimension stratégique. En effet, ces discours ont bien vocation à stimuler la participation des habitants, laquelle servirait à pallier certains problèmes de gestion publique. Si cet objectif est revendiqué par toutes les directions des services communication, les propos de la directrice de la communication de Poissy, qui décrit le dispositif comme un palliatif du manque de places de crèches dans sa ville, sont à ce titre assez emblématiques :

"Mettre ce service était une manière d'inciter les gens à se débrouiller par le privé [...]. Avec la nouvelle équipe, ce service rentre dans le cadre de ce que l'on veut faire [...]. C'est pour répondre aux besoins des habitants d'avoir une réponse rapide sur les gardes d'enfants » (Directrice de la communication, Poissy)

Par voie de conséquence, ces enjeux liés à la gestion publique locale renforcent la position du responsable de communication vis-à-vis du politique, puisque cette gestion publique locale, pour être " efficace ", peut reposer davantage sur des logiques communicationnelles. Cette ambition de la profession, consistant à faire évoluer le métier d'une fonction d'exécution à une fonction stratégique, n'est pas nouvelle si l'on prend notamment le cas des sondages d'opinion (Fourdin 1994:83), mais elle est renouvelée avec ces nouveaux dispositifs.

\section{Conclusion}

L'adoption d'une plateforme numérique de services par une ville a pour corollaires de renouveler les thèmes de la communication territoriale, de modifier les pratiques professionnelles des responsables de communication des collectivités, leurs rapports avec les élus locaux, et par extension les liens entre la communication publique et la communication politique qui s'étaient instaurés depuis les lois de décentralisation de 1982. Néanmoins, cette action publique renouvelle les cadres de la participation citoyenne et s’inscrit dans le prolongement du paradigme de la proximité, qui « est fondée 
comme sur un jeu d'équivalences naturalisées dont l'effet symbolique est puissant : proximité=implication=participation=efficacité=légitimité » (Le Bart et Lefebvre 2005:13). Pour répondre à ces enjeux, les communicants territoriaux ont pour tâches : d'une part, de mobiliser les acteurs du territoire censés eux-mêmes participer activement à la « co-production » de la nouvelle offre de services de la ville, puisqu'il est communément admis que sans une activité d'animation des acteurs, les plateformes de co-innovation ne peuvent émerger (Lelong et Gayoso 2010:122), et d'autre part, de faire connaître ces nouveaux services « centrés sur l'usager ». La visée opérationnelle de la communication territoriale accompagnant ces dispositifs n'est pas sans faire écho aux nombreuses initiatives territoriales françaises qui émergent depuis 2010 autour du mouvement " open data ", s'inscrivant également dans la lignée du « nouveau management public ». Dans ces dernières initiatives plus encore, les responsables de communication territoriale occupent une position centrale dans cette politique de transformation des services publics reposant sur la participation active d'acteurs tiers, lesquels deviennent responsables de la création de nouveaux services contextualisés de première nécessité, répondant à des besoins premiers « ici » et « maintenant » (Labelle, Le Corf, 2012).

\section{BIBLIOGRAPHIE}

BACQUÉ M.-H., REY H., SINTOMER Y., «Introduction. La démocratie participative, un nouveau paradigme de l'action publique? ", dans BACQUÉ M.-H., REY H. Gestion de proximité et démocratie participative, Paris, La Découverte, 2005, p. 9-46.

BARABEL M., MAYOL S., MEIER O., «Les médias sociaux au service du marketing territorial : une approche exploratoire », Management $\Xi$ Avenir, 2010, n³2, p. 233-253.

BLONDIAUX L., Le nouvel esprit de la démocratie, Paris, Seuil, 2008.

BOLTANSKI L., CHIAPELlO È., Le nouvel esprit du capitalisme, Paris, Gallimard, 1999.

CORMERAIS F., «Territoires et innovation, entre valeur sociétale et développement des capabilités ", Cabiers du CERCI, 2009, nº, p. 259-271.

FOURDIN M., «La professionnalisation de la communication locale : un paradoxe? », Réseaux, 1994, vol. 12, n64, p. 75-89.

KRZĄTAŁA-JAWORSKA E., "Comprendre la diversité de la participation politique en ligne. Une étude comparative de Facebook et ComunaliMilano2011.it ", communication au colloque "IPSA's XXIInd World Congress of Political Science ", Madrid, 9 juillet 2012.

LABELLE S., LE CORF J.-B., "Opendata as new commitment of governments: from injunction of transparency to coproduction of services », communication au colloque «IPSA’s XXIInd World Congress of Political Science », Madrid, 11 juillet 2012. 
LAVIGNE A., "Suggestion d'une modélisation de la communication publique : principales formes discursives et exemples de pratiques ", Les Cahiers du journalisme, 2008, n'18, p. 232-245.

LE BART C. « Les bulletins municipaux : une contribution ambiguë à la démocratie locale », Hermès, 2000, n²6.

LE BART C., LEFEBVRE R., " Introduction. Une nouvelle grandeur politique? ", dans LE BART C., LEFEBVRE R., La proximité en politique, Rennes, PUR, 2005, p. 11-30.

LE CORF J.-B., « Services numériques et qualité de vie: le positionnement stratégique d'une entreprise web vis-à-vis des collectivités territoriales », dans DELAVAUD G. (dir.). Nouveaux territoires médiatiques, Rennes, Apogée, 2012, à paraître.

LE CORF J.-B., " La création collective de la plateforme de services publics numériques de la rive droite de Bordeaux : entre communication territoriale et gestion publique locale », Études de communication, 2011, n³7, p. 131-147.

LELONG B., GAYOSO É., « Innovation avec l'usager et plateformes collaboratives », Réseaux, 2010, n¹64, p. 97-126.

MATTELART A., La globalisation de la surveillance : aux origines de l'ordre sécuritaire, Paris, la Découverte, 2007.

MUSSO P., « La raison du Réseau », Quaderni, 2003, n52, p. 55-66.

NEVEU C., "Une "petite fabrique de territoire" : quartiers et citoyenneté à Roubaix », Ethnologie française, 2004, vol. 34, n¹, p. 59-66.

NONJON M., "Les usages de la proximité dans le champ participatif ", dans LE BART C., LEFEBVRE R., La proximité en politique, Rennes, PUR, 2005, p.169-181.

OGIEN A., " La perfection gestionnaire: rhétorique de l'efficacité et démocratie ", dans OLLIVIER-YANIV C., RINN M., Communication de l'État et gouvernement du social, Grenoble, PUG, 2009, p. 49-66.

OLLIVER-YANIV C., "Avant-propos ", dossier "Secret et pouvoir : les faux-semblants de la transparence », Quaderni, 2003, vol. 52, n52 p. 39-41.

PAILLIART I., «Territoires, identités et communication », dans OLIVESI S., Sciences de l'information et de la communication, Grenoble, PUG, 2006, p. 113-128.

PAILLIART I., "Les enjeux locaux de la démocratie électronique », Hermès, 2000, p. 26-27.

SINTOMER Y., « Du savoir d'usage au métier de citoyen ? ”, Raisons politiques, 2008, n'31, p. 115-133.

Résumé : Nous étudions le cas d'une plateforme d'échange de services entre habitants, rattachée à différents sites Internet municipaux. A partir de l'étude de neuf villes mettant en place une même plateforme, notre approche vise à observer les pratiques des responsables de communication des villes pour comprendre comment celles-ci sont affectées par l'introduction du dispositif. Notre propos s'appuie sur une analyse thématique d'entretiens semi-directifs menés auprès de responsables de communication, une analyse des sites des municipalités mettant en place cette plateforme, ainsi que l'étude d'un corpus d'articles portant sur le service, parus dans leurs bulletins municipaux. 
Nous articulons notre démonstration en trois temps pour montrer d'abord comment les communicants publics cherchent à garantir une information sur le nouveau service offert, ensuite comment ces professionnels conservent une autonomie relativement limitée vis-à-vis de l'instance politique, et enfin leur nouvelle position de stratège qui découle de l'adoption de cette plateforme, entraînant une visée plus opérationnelle de la communication territoriale dans sa prétention à mobiliser les acteurs du territoire afin de moderniser l'offre de services aux habitants.

Mots-clés : communicant public, collectivités territoriales, plateformes collaboratives, communication publique, communication politique.

Abstract : Within the framework of this article, we shall study the case of a platform of
exchange of services between inhabitants, connected with various municipal city web sites. From
the study of nine cities setting up the same platform, our approach aims at observing the practices
of the heads of communications of cities. We press our comment on the results of the analysis of
conversations with heads of communications, the analysis of the web sites of municipalities
setting up this platform and the analysis of a corpus of articles concerning the service of the
various cities. At first, we show how the public communicators try to guarantee an information
about the new offered service. Secondly, we analyze how the professionals of the communications
offices of cities keep an autonomy relatively limited towards the political authority. In the third
time, we are interested in the extension of the skills of the head of communications, beyond the
technical aspects, and in his strategist's new position.

Keywords : Communicator public, Collaboratives platforms, Public communication, Political communication. 